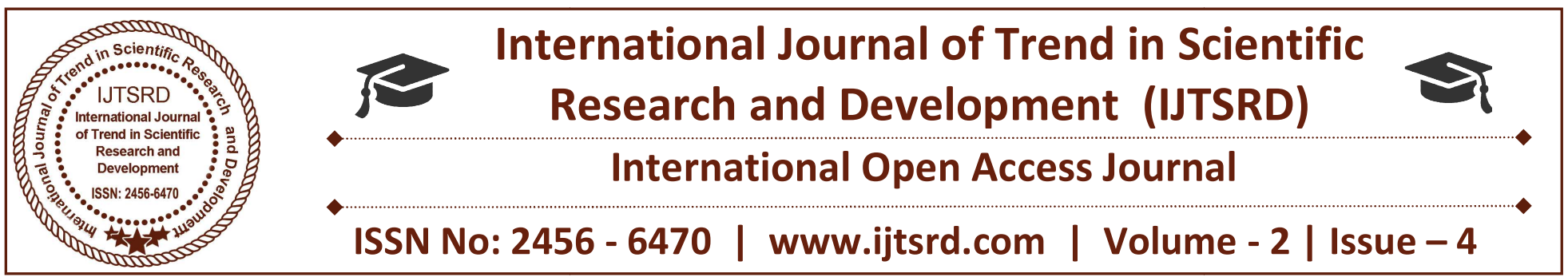

\title{
Biosynthesis of Silver (Ag) Nanoparticles using Plant Derivatives of Delonix elata
}

\author{
K. Vinoth Kumar \\ Department of Environmental Sciences, \\ Tamil Nadu Agricultural University, Coimbatore, Tamil Nadu, India
}

\begin{abstract}
Nanoparticles are being viewed as fundamental building blocks of nanotechnology. The development of new chemical or physical methods, the concern for environmental contaminations are also heightened as the chemical procedures involved in the synthesis of nanoparticles generate a large amount of hazardous byproducts. Thus, there is a need for green chemistry that includes a clean, non toxic and environment friendly method of nanoparticles synthesis. As a result, researchers in the field of nanoparticles synthesis and assembly have turned to biological system of inspiration. One of them is the synthesis of nanoparticles using plant leaf extracts eliminating the elaborate process of marinating the microbial culture and often found to be kinetically favourable than other bioprocesses. The present study deals with the synthesis of silver nanoparticles using leaf extract of Delonix elata. The synthesized silver nanoparticles were predominately spherical in shape, polydispersed and ranged in size from 20-60 $\mathrm{nm}$.
\end{abstract}

Keywords: Green approach, Biosynthesis, Delonix elata, Ag nanoparticles

\section{INTRODUCTION}

Nanoparticles are being viewed as fundamental building blocks of nanotechnology. An important aspect of nanotechnology concerns the development of experimental processes for the synthesis of nanoparticles of different sizes, shape and controlled dispersity. With the development of new chemical or physical methods, the concern for environmental contaminations are also heightened as the chemical procedures involved in the synthesis of nanoparticles generate a large amount of hazardous byproducts. Thus, there is a need for green chemistry that includes a clean, non toxic and environment friendly method of nanoparticles synthesis. As a result, researchers in the field of nanoparticles synthesis and assembly have turned to biological system of inspiration. Among the biological system plants have found application particularly in metal nanoparticles synthesis. Use of plants for synthesis of nanoparticles could be advantageous over other environmentally benign biological processes as this eliminates the elaborate process 0 maintaining cell culture. Biosynthetic processes for nanoparticles would be more useful if nanoparticles were produced extracelluarly using plants or their extracts and in a controlled manner according to their size, shape and dispersity (Kumar and Yadav, 2008).

Biosynthesis of nanoparticles by plant extracts is currently under exploitation. The aqueous silver nitrate solution, after reacting with geranium (Pelargonium graveolens) leaf extract, led to rapid formation of highly stable, crystalline silver nanoparticles (16 to $40 \mathrm{~nm}$ ) (Shankar et al., 2003). Silver nanoparticles were synthesized by treating silver ions with Capsicum annuum L. leaf extract, the crystalline phase of the nanoparticles changed from polycrystalline to single crystalline and their size increased with increasing reaction time. Five hours reaction time led to spherical and polycrystalline shaped nanoparticles $(10 \pm 2 \mathrm{~nm})$ (Li et al., 2007). In this paper, we report on the biosynthesis of pure metallic nanoparticles of silver by the reduction of aqueous $\mathrm{Ag}^{+}$ions with the leaf extract of Delonix elata. 


\section{MATERIALS AND METHODS}

\section{Preparation of leaf extract}

The fresh and young leaf samples of Delonix elata was collected and washed thoroughly with sterile double distilled water (DDW) and finally surface sterilized with $0.1 \% \mathrm{HgCl}_{2}$ for 2 - 3 min under the hood of laminar air flow. Twenty gram of sterilized leaf samples were taken and cut into small pieces. Finely cut leaves were placed in a $500 \mathrm{ml}$ Erlenmeyer flask containing $100 \mathrm{ml}$ of sterile DDW. After that the mixture was boiled for $5 \mathrm{~min}$ and filtered. The extract was stored in $4{ }^{0} \mathrm{C}$.

\section{Synthesis of silver nanoparticles}

Silver nitrate was used as precursor of synthesizing the silver nanoparticles. Five $\mathrm{ml}$ of leaf extract was added to $100 \mathrm{ml}$ of $1 \mathrm{mM} \mathrm{AgNO}_{3}(99.99 \%)$ aqueous solution in conical flask of $250 \mathrm{ml}$ content at room temperature. The flask was thereafter put into shaker $(150 \mathrm{rpm})$ at $30^{\circ} \mathrm{C}$ and reaction was carried out for a period of $48 \mathrm{~h}$.

\section{$U V$-visible spectroscopy analysis}

The colour change in reaction mixture was recorded through visual observation. The bioreduction of silver ions in aqueous solution was monitored by periodic sampling of aliquot $(1 \mathrm{ml})$ and subsequently measuring UV-vis spectra of the solution. UV-vis spectra of these aliquot was monitored as a function of time of reaction on Elico UV-vis spectrophotometer (model S3-159) operated at a resolution of $1 \mathrm{~nm}$.

\section{XRD measurement}

The sample was drop-coated onto aluminum plate by just dropping a small amount of sample on the plate frequently, allowed to dry and finally thick coat of sample on plate was prepared. The XRD measurement was performed on a Shimazdu, model LabX-XRD6000 instrument operated at a voltage of 20 to $30 \mathrm{keV}$ and a current of $30 \mathrm{~mA}$ with $\mathrm{Cu} \mathrm{K} \alpha$ radiation with a wavelength of $1.5418 \AA$.

\section{Determination of crystalline size}

Average crystallite size of silver was calculated using the Scherrer's formula,

$$
\mathrm{D}=\mathrm{k} \lambda / \beta \cos \theta
$$

D- Average crystallite size: K- Constant: $\lambda$ - X-ray Wavelength: $\beta$ - Angular FWHM of the XRD peak at the diffraction angle: $\theta$ - Diffraction angle.

\section{SEM analysis}

The thin film of the samples were prepared on a small aluminum plate by just dropping a very small amount of the sample on the plate, extra solution were removed using a blotting paper and then the film on the plate was allowed to dry for overnight. The SEM analysis was performed on a JEOL, model JSM-6390 instrument operated at an accelerating voltage of $20 \mathrm{keV}$ and counting time of $100 \mathrm{~s}$.

\section{FT-IR measurement}

FT-IR measurement of sample was performed using the Nicolet Avatar Model FT-IR spectrophotometer in a diffuse reflectance mode at a resolution of $4 \mathrm{~cm}^{-1}$ in $\mathrm{KBr}$ pellets.

\section{RESULT AND DISCUSSION}

The extracellular synthesis of silver nanoparticles occurred during the exposure of Delonix elata leaf extract to $1 \mathrm{mM}$ aqueous silver nitrate solution. The complete reduction of silver ions was observed after $48 \mathrm{~h}$ of reaction at $30^{\circ} \mathrm{C}$ under shaking condition. The colour change in reaction mixture was observed during the incubation period, because the formation of silver nanoparticles is able to produce particular colour in the reaction mixtures due to their specific properties. The appearance of dark yellowish-brown colour is a clear indication of the formation of silver nanoparticles in the reaction mixture (fig.1). The colour exhibited by metallic nanoparticles is due to the coherent excitation of all the "free" electrons within the conduction band, leading to an in-phase oscillation and is known as Surface Plasmon Resonance-SPR (Akanna et al., 2010).

UV-vis spectroscopy analysis showed that the SPR absorbance band of silver nanoparticles synthesized using Delonix elata leaf extract centered at $448 \mathrm{~nm}$ (fig 2.) and steadily increases in intensity as a function of time of reaction without any shift in the peak wavelength. The frequency and width of the surface plasmon absorption depends on the size and shape of the metal nanoparticles as well as on the dielectric constant of the metal itself and the surrounding medium (Mukherjee et al., 2002). XRD pattern obtained for silver nanoparticles showed characteristic peaks near the $2 \theta$ value of $38.76^{\circ}$ (fig.3). A Bragg 
reflection corresponding to the (111) sets of lattice planes are observed which may be indexed based on the face-centered cubic (fcc) structure of silver (Dubey et al., 2009). The XRD pattern thus clearly shows that the silver nanoparticles are crystalline in nature. In addition to the Bragg peak representative of fcc silver nanocrystals, additional and yet unassigned peaks were also observed suggesting that the crystallization of bio-organic phase occurs on the surface of the silver nanoparticles (Sathyavathi et al., 2010).

Crystallite size of silver nanoparticles as estimated from the Full width at half maximum (FWHM) of the (111) peak of silver using the Scherrer's formula exhibited average particles size of $22 \mathrm{~nm}$.

\section{Crystalline size of synthesized silver nanoparticles}

\begin{tabular}{|c|c|c|c|c|c|}
\hline Plant extract & $\begin{array}{c}\boldsymbol{\theta} \text { value } \\
\text { [degree] }\end{array}$ & $\begin{array}{c}\text { d- spacing } \\
{[\AA \mathbf{A}]}\end{array}$ & $\begin{array}{c}\text { FWHM } \\
\text { [degree] }\end{array}$ & $\begin{array}{c}\text { Intensity } \\
\text { [CPS] }\end{array}$ & $\begin{array}{c}\text { Average } \\
\text { Particle } \\
\text { size }[\mathbf{n m}]\end{array}$ \\
\hline Delonix elata & 19.38 & 2.321 & 0.703 & 33.0 & 21.83 \\
\hline
\end{tabular}

SEM image has shown individual silver particles as well as a number of aggregates. The morphology of the silver nanoparticles was predominately spherical and aggregated into larger irregular structure with no well-defined morphology observed in the micrograph (fig.4). The nanoparticles were not in direct contact even within the aggregates, indicating stabilization of the nanoparticles by a capping agent (proteins secreted by plant leaf extracts). The presence of secondary materials capping with the esilver nanoparticles and this capping may be assigned to bio-organic compounds from leaf extracts (Rajesh et al., 2009).

Fourier Transform Infra-Red (FT-IR) spectroscopy analysis showed that the synthesized silver nanoparticles are capped with biomolecule compounds which are responsible for reduction of silver ions. The wavenumber or frequency $\left(\mathrm{cm}^{-1}\right)$ of absorption band or peak assigned to the type of vibration, intensity and functional groups of the silver nanoparticles synthesized using Delonix elata leaf extract are shown in fig 5.Different functional groups were involved in reduction of silver ions to silver nanoparticles. The peaks in the region of 3400 to 3200 $\mathrm{cm}^{-1}$ and 3000 to $2850 \mathrm{~cm}^{-1}$ were assigned to $\mathrm{O}-\mathrm{H}$ stretching of alcohol and phenol compounds and aldehydic -C-H- stretching of alkanes, respectively. The peaks in the region of 1640 to $1550 \mathrm{~cm}^{-1}$ and 1450 to $1375 \mathrm{~cm}^{-1}$ correspond to N-H (bend) of primary and secondary amides and $\mathrm{C}-\mathrm{H}\left(-\mathrm{CH}_{3}-\right.$ bend) of alkanes, respectively. The peaks at the region of 1350 to $1000 \mathrm{~cm}^{-1}$ correspond to $-\mathrm{C}-\mathrm{N}$ - stretching vibration of the amine or -C-O- stretching of alcohols, ethers, carboxylic acids, esters and anhydrides. FT-IR analysis reveals that the carbonyl group from amino acid residues and proteins has the stronger ability to bind metal indicating that the proteins could possibly form a layer covering the metal nanoparticles (i.e., capping of silver nanoparticles) to prevent agglomeration and thereby stabilize the medium. This suggests that the biological molecules could possibly perform dual functions of formation and stabilization of silver nanoparticles in the aqueous medium (Sathyavathi et al., 2010).

\section{CONCLUSION}

Delonix elata capable of producing silver nanoparticles extracellularly and the synthesized silver nanoparticles are quite stable in solution. The control of shape and size of silver nanoparticles seems to be easy with the use of plant leaf extracts. The synthetic methods based on naturally occurring biomaterials provide an alternative means for obtaining the nanoparticles. Use of plants in synthesis of nanoparticles is quite novel leading to truly 'green chemistry' route. This green chemistry approach towards the synthesis of nanoparticles has many advantages such as, ease with which the process can be scaled up, economic viability, eco-friendly and safe way to produce nanoparticles.

\section{REFERENCES}

1. Kumar V. and Yadav S.K. (2008). Plant-mediated synthesis of silver and gold nanoparticles and their applications, J. Chem. Technol. Biotechnol., 1, 17. 
2. Shankar S.S., Ahmad A., Pasricha R. and Sastry M. (2003). Bioreduction of chloroaurate ions by geranium leaves and its endophytic fungus yields gold nanoparticles of different shapes. J. Mater. Chem., 13, 1822-1826.

3. Li S., Shen Y., Xie A., Yu X., Qiu L., Zhang L. and Zhang Q. (2007). Green synthesis of silver nanoparticles using Capsicum annuum L. extract, Green Chem., 9, 852-858.

4. Akanna S., Prasad K.V., Elumalai E.K. and Savithramma N. (2010). Production of biogenic silver nanoparticles using Boswellia ovalifoliolata stem bark, Digest J. Nanomat. Biostruct., 5(2), 369-372.

5. Mukherjee P., Senapati S., Mandal D., Ahmad A., Khan M.I., Kumar R. and Sastry M. (2002). Extracellular synthesis of gold nanoparticles by the fungus: Fusarium oxysporum. Chem. Bio. Chem., 3 (5), 461-463.

6. Dubey M., Bhadauria S. and Kushwah B.S. (2009). Green synthesis of nanosilver particles from extract of Eucalyptus hybrinda leaf. Digest J. Nanomat. Biostruct., 4(3), 537-543.

7. Sathyavathi R., Krishna M. B., Rao S.V., Saritha R. and Rao D.N. (2010). Biosynthesis of silver nanoparticles using Coriandrum sativum leaf extract and their application in nonlinear optics. Adv. Sci. Lett., 3, 1-6.

8. Rajesh W.R., Jaya R.L., Niranjan S.K., Vijay D.M. and Sahebrao B.K. (2009). Phytosynthesis of silver nanoparticles using Gliricidia sepium. Curr. Nano Sci., 5, 117-122

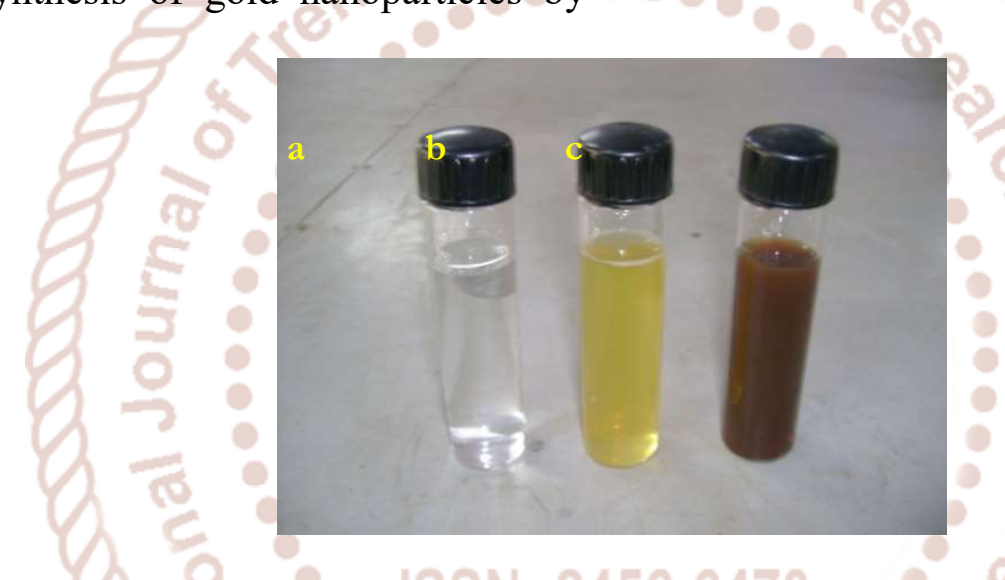

Fig 1. Optical photograph of (a) $1 \mathrm{mM} \mathrm{AgNO}_{3}$ solution (b) Leaf extract (c) Leaf extract $+\mathrm{AgNO}_{3}$ after $48 \mathrm{~h}$ of reaction

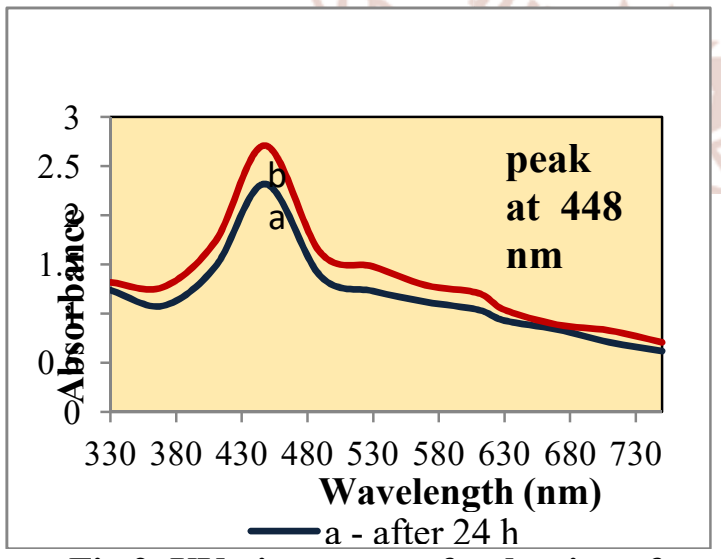

Fig 2. UV-vis spectra of reduction of Ag ions to Ag nanoparticles

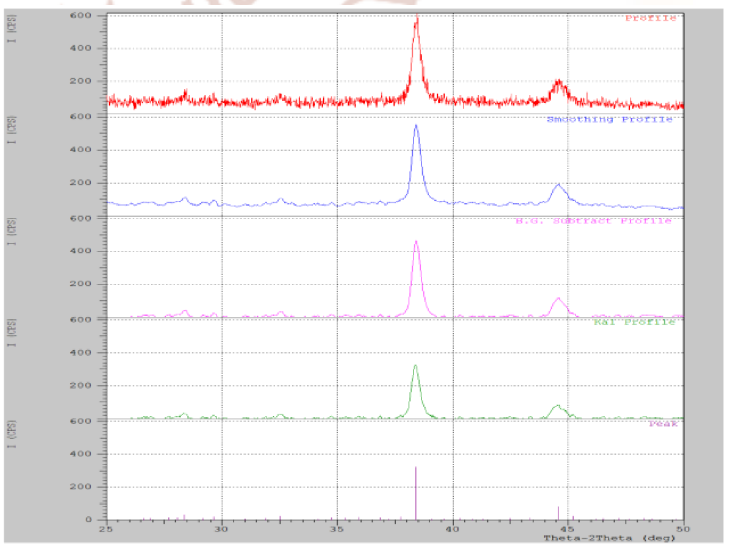

Fig 3. XRD pattern of Ag nanoparticles 
International Journal of Trend in Scientific Research and Development (IJTSRD) ISSN: 2456-6470

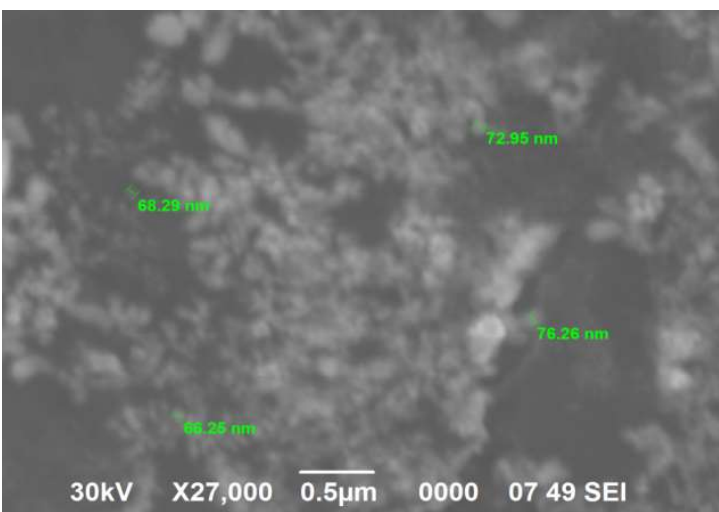

Fig 4. SEM image of Ag nanoparticles Ag nanoparticles

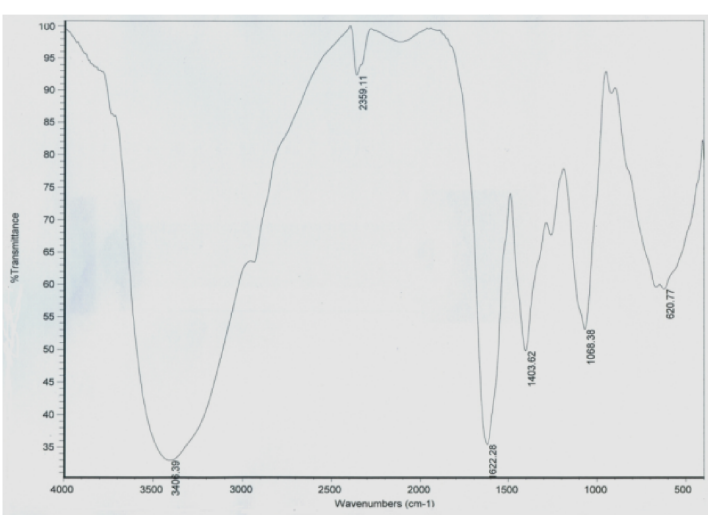

Fig 5. FT-IR spectrum of

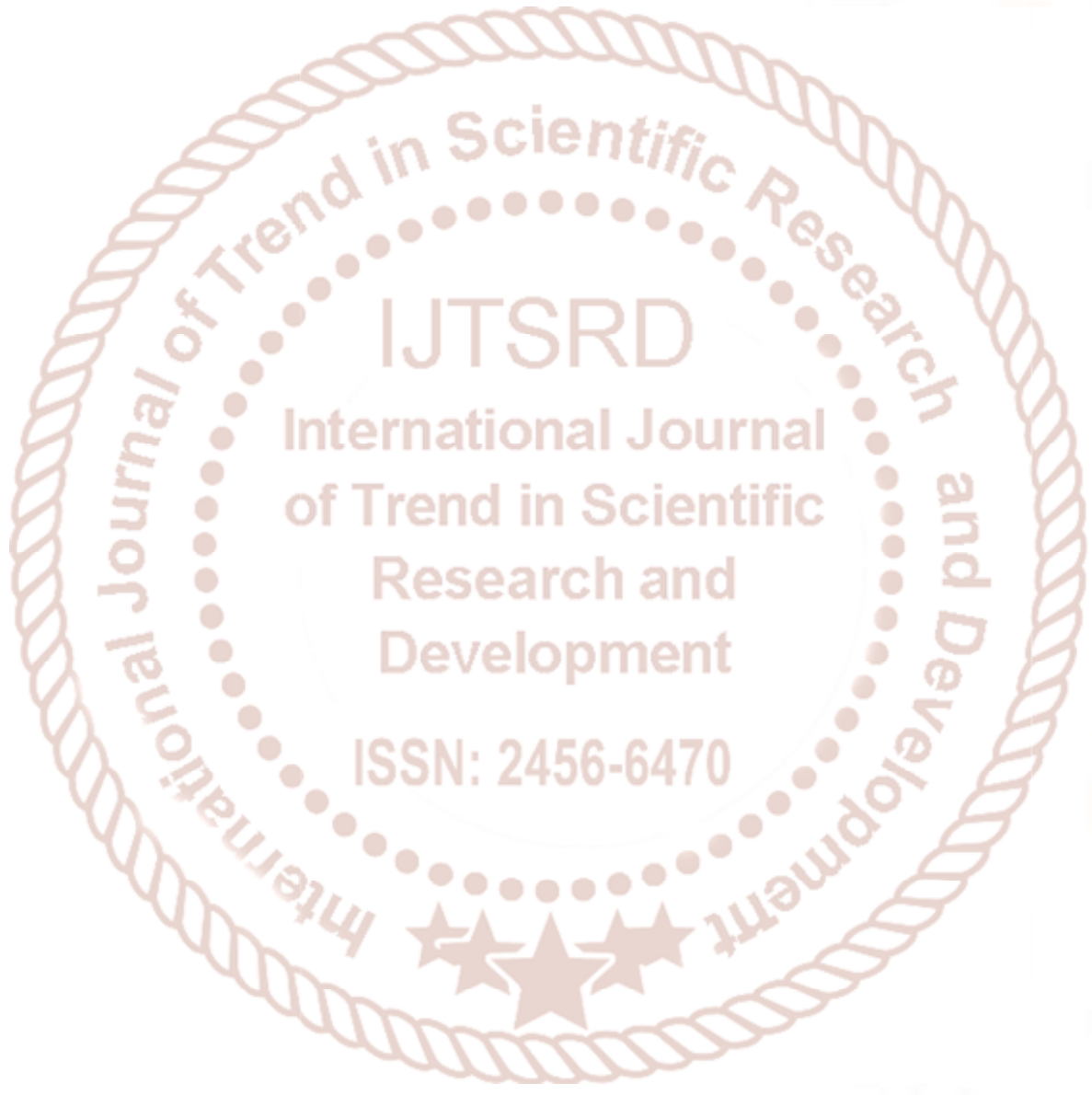

